\title{
Modelling sea level and East Australian Current co-variability using the Hilbert-Huang transform
}

\author{
F.M. Ziaeyan Bahri $^{\text {a }}$, J.J. Sharples ${ }^{\text {a }}$, X.H. Wang ${ }^{\mathrm{a}}$ and Y.-J. Sun ${ }^{\text {a }}$ \\ ${ }^{a}$ School of Physical, Environmental and Mathematical Sciences, UNSW Canberra, Australia
}

Email: Fatemeh.Ziaeyanbahri@student.adfa.edu.au

\begin{abstract}
Sea-level rise is one of the most important consequences of global warming, and has global and regional im-pacts. Studying changes in sea level on a regional scale is essential to the development of morereliable coastal adaptation plans. On a regional scale, there is a superimposition of a number of factors, which complicates the analysis: oceanographic data are usually non-stationary and non-linear.

In this study we use the Empirical Mode Decomposition (EMD)/Hilbert-Huang Transform (HHT) method to analyse East Australian Current (EAC) transport and sea level data from Fort Denison, Sydney. The focus of this study is the long-term time scale consequences of climate change. The results show a rise in sea level of about $1.2 \mathrm{~mm} / \mathrm{yr}$ over 98 years, and an increase in the total EAC transport in the Sydney region of about $8.75 \mathrm{~Sv}$ in over 61 years. These preliminary results also indicate a statistically significant ( 7 years) lagged correlation of $r=0.84$ ( $\mathrm{p}$ value $<10^{-4}$ ) between the multidecadal variability in EAC and sea level. However, it is possible that this apparent correlation may in some part be an artifact of the modelling methodology.

Nevertheless, the EMD/HHT methodology provides a way of decomposing non-stationary data into various intrinsic modes, which can be compared with the known behaviour of various physical drivers of sea level variability. Moreover, it allows comparison of different aspects of the ocean system and can be used to infer the existence of interrelationships, which can be used to improve understanding of local ocean dynamics. These aspects of the methodology are explored in the paper.
\end{abstract}

Keywords: Sea level rise, East Australian Currents, regional sea level rise, Hilbert-Huang Transform, empirical mode decomposition 
F.M. Ziaeyan Bahri et al. , Modelling sea level and EAC co-variability using HHT...

\section{INTRODUCTION}

Changes in sea level have important consequences for coastal regions. To facilitate more-reliable local adaptation planning, it is necessary to have a better understanding of sea-level variability at regional scales (Rahmstorf, 2012b). However, the study of regional sea-level variability is complicated by a number of factors, including the bathymetric differences between regions, variations in ocean currents, and combinations of these effects. As a consequence, obtaining a comprehensive understanding of regional sea-level variability is a difficult, multi-faceted problem.

Levermann et al. (2005) investigated the relationship between thermohaline circulation, and the local and global variability of sea level. They found that local sea level can rise by up to $1 \mathrm{~m}$ due to local current circulation variability. Changes in sea level due to regional variations can be as large as the climatic signal on multi-decadal time scales (Rahmstorf, 2012b,a).

Several studies have analysed the spatial variability in sea level, especially in the Atlantic Ocean. Ezer et al. (2013) found that sea level in the mid-Atlantic rose $1 \mathrm{~cm}$ from 2000 to 2011. They also found a strong relationship between the changes in the Gulf Stream, and sea-level variability, and demonstrated that changes in the Gulf Stream due to climate change have contributed significantly to sea-level rise in the region.

Long-term trends in sea-level variability in the Australian region are consistent with the effects of climate change (Hughes, 2003; Mitchell et al., 2000). The average change in sea level was found to be about $+0.3 \mathrm{~mm}$ per year, which is less than the IPCC global estimate (Mitchell et al., 2000). However, few studies have considered the influence of local ocean currents on regional sea-level variability. Holbrook et al. (2011) used satellite altimeter data to estimate large-scale sea-level changes at Fort Denison, Sydney. They cited Rossby waves as a connecting mechanism between the variations of ocean-adjusted decadal trends in sea level and the East Australian Current transport, using Fort Denison tidal data. They showed that there is a strong relationship between monthly sea-level variability and the Inter-decadal Pacific Oscillation (IPO) index, with a correlation coefficient of $r=0.65$, significant at the $95 \%$ level. The correlation was found to be weaker during El Nino events.

In the east Australian region, the East Australian Current (EAC) is the dominant west boundary current. Changes in the EAC can affect the region's climate and ocean dynamics. Ridgway et al. (2008) found that penetration of the EAC has increased southward in the last 60 years. As a consequence the region has become warmer and saltier, with mean trends of $+2.28^{\circ} \mathrm{C}$ per century, and +0.34 psu per century over the period 1994-2002, respectively. Cetina-Heredia et al. (2014) showed furthermore that the point of separation of the EAC from the coast has also migrated southward in the last couple of decades. Considering the EAC as a wind-driven western boundary current, it is expected that variability in wind patterns will have a strong impact on EAC transport.

Cai (2006) used the Godfreys Island Rule model, a re-driving transport model, to show that changes in Antarctic ozone will change the surface wind stress, and thereby affect the large-scale circulation in the Southern Hemisphere. Large-scale circulation variations will strengthen the EAC by up to $20 \%$, and perhaps even more in the south. At $20-30^{\circ} \mathrm{S}$, the EAC has decreased, whereas beyond $30^{\circ} \mathrm{S}$ it has increased by about $9 \mathrm{~Sv}$, or $20 \%$, over the last decade. According to Cai (2006), the EAC is becoming warmer and saltier in the south, with implications for local marine ecosystems.

In this study we follow the work of Ezer et al. (2013) and investigate links between changes in the EAC transport and sea-level variability along the east Australian coast by considering their co-variability at the latitude of Fort Denison. Tidal-gauge data is used to provide information on sea level and the output of a numerical ocean circulation model to provide information on EAC transport. The Hilbert-Huang transform (HHT) is used to decompose the sea-level and EAC time series to examine co-variability at multiple time scales.

\section{Data AND Methods}

\subsection{Sea-level and EAC transport data}

Monthly mean tidal-gauge data recorded at Fort Denison $\left(33.8^{\circ} \mathrm{S}\right)$ is used to determine sea level at Sydney. Fort Denison has the longest sea-level record in Australia, spanning the years 1886 to 2012. The data used in this study was obtained from the Permanent Service of Mean Sea Level website (http://www.psmsl.org/data/obtaining/map.html). 
The EAC is a western boundary current that moves southward from the equator along the east Australian coast, before it separates towards the east into the Tasman Sea. The separation point changes in latitude over time. The EAC can separate from the coast anywhere between $30^{\circ} \mathrm{S}$ and $34^{\circ} \mathrm{S}$, with the region near the separation point usually dominated by eddies (Cetina-Heredia et al., 2014). Here, the geostrophic current transport has been extracted from the Ocean General Circulation Model for the Earth System (OFES). OFES is a global high resolution ocean-only model pinoeered by Masumoto et al. (2004) and Sasaki et al. (2008). The accuracy of this simulator was tested by Sebille et al. (2012), who compared OFES simulated data with altimeter data and demonstrate a high degree of agreement. The horizontal resolution in OFES model is 0.1 degrees, with 54 vertical levels. The model domain covers $75^{\circ} \mathrm{S}$ to $75^{\circ} \mathrm{N}$ (Masumoto et al., 2004). In this study we used geostrophic transport of the EAC at $33.85^{\circ} \mathrm{S}$, the latitude of Fort Denison. The simulation was conducted from 1950 to 2010, from the surface to the bottom, and up to $100 \mathrm{~km}$ from the coast. In this study, the total EAC transport (including eddies) was used.

\subsection{Empirical mode decomposition/ Hilbert-Huang Transform}

Several studies have been conducted in Australia using Fourier-based methods to investigate sea-level variability (e.g. Hughes, 2003; Mitchell et al., 2000). However, due to the non-stationary and non-linear nature of sea-level data, these studies are of limited validity. To overcome these issues, the Hilbert-Huang Transform (HHT) method was developed to analyse non-stationary and non-linear time series, and has been widely used in geophysical studies. For example, Ezer et al. (2013) used the Empirical Mode Decomposition (EMD) and the HHT to study regional sea-level change along the mid-Atlantic coast, and its correlation with Gulf Stream transport. They reported a strong correlation between sea-level variability and variations in the Gulf Stream.

The EMD procedure decomposes a time series $y(t)$ into a number of intrinsic mode functions (IMFs), which satisfy certain defining conditions (Huang et al., 1998). The IMFs of $y(t)$ are determined through a sifting process that first identifies all the local extrema in the signal $y(t)$. The local maxima and local minima are then interpolated separately to define upper and lower envelopes of $y$. The mean of the upper and lower envelopes $m_{1}$ is then subtracted from the original time series $y$ to give the first protomode $h_{1}=y-m_{1}$. This procedure is then repeated in an iterative manner, so at the $j$ th iteration we have $h_{j}=h_{j-1}-m_{j}$, where $m_{j}$ is the mean of the upper and lower envelopes of $h_{j-1}$, determined by interpolation of the local maxima and minima. The iteration is continued $k$ times until a stoppage condition is satisfied (Huang and $\mathrm{Wu}, 2008$ ). The time series $h_{k}$ is identified as the first IMF, denoted $c_{1}$. These functions should satisfy the following conditions: (1) the number of extrema and the number of zero crossings in the whole data set must either be equal or differ by at most 1 ; (2) at any data point, the mean value of the envelope defined using the local maxima and the envelope defined using the local minima is zero (Huang and $\mathrm{Wu}, 2008$ ).

This process is then applied to the residual time series $r_{1}=y-c_{1}$ to deliver the second IMF $c_{2}$ and the second residual $r_{2}=r_{1}-c_{2}$. Subsequent IMFs $c_{3}, \ldots, c_{n}$ and residuals $r_{3}, \ldots, r_{n}$ are obtained similarly. The sifting process terminates when $r_{n}$ has at most one extremum. The original time series $y(t)$ has then been decomposed as:

$$
y(t)=\sum_{j=1}^{n} c_{j}(t)+r_{n}(t) .
$$

For a more detailed exposition of the EMD procedure, the reader is referred to Huang et al. (1998) and Huang and $\mathrm{Wu}$ (2008). Unlike global methods like the Fourier transform, the EMD procedure expresses the input time series in terms of an a posteriori defined basis which is derived from the data itself. When combined with the Hilbert transform (Hahn, 1996), which delivers information about the frequency/power spectra of the intrinsic modes, EMD constitutes a powerful data-analysis technique that can be applied to nonlinear and nonstationary time series (Schlurmann, 2002). The combination of EMD and Hilbert spectral analysis is referred to collectively as the Hilbert-Huang Transform. See Ezer et al. (2013, Fig. 4) for an example of its use in oceanographic studies.

\section{Results}

In this study, we first considere spatial sea-level variability along the east coast over the last 30 years. Then, the EMD/HHT method is used to investigate the co-variability of sea level and the East Australian Current transport at latitude of Fort Denison. The sea-level data are used to obtain the total trend from 1880 to 2013. 
F.M. Ziaeyan Bahri et al. , Modelling sea level and EAC co-variability using HHT...

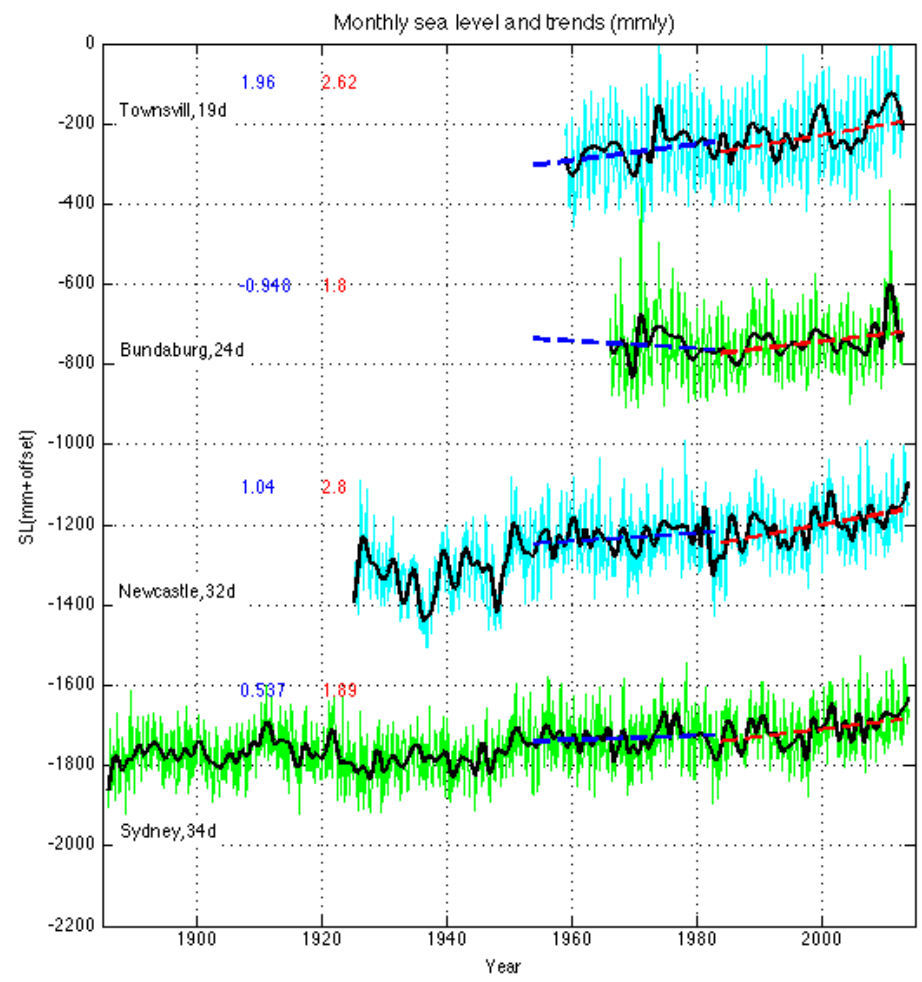

Figure 1. Sea-level variability along the east coast of Australia. Solid green and light blue line is the observation sea level data. Solid black curves is sub-decadal variation in sea level. Dashed blue line is linear regression over 30 years time period from 1953 to 1983 and dashed red line is linear regression from 1983 to 2013.

However, due to the limited span of the EAC transport data (1950 to 2010), we consider sea-level variability over the same time period.

\subsection{Acceleration in sea-level rise}

Previous studies have shown that sea level has been rising over the last couple of decades in Australia (Church et al., 2006). However, how fast the sea level is rising or the relationship with local ocean dynamics has received less attention in the literature. Figure 1 illustrates sea-level variability at four stations along the east coast of Australia (Townsville, Bundaberg, Newcastle and Sydney, respectively $19^{\circ} \mathrm{S}, 24^{\circ} \mathrm{S}, 32^{\circ} \mathrm{S}$ and $34^{\circ} \mathrm{S}$ ). The black solid curves represent sub-decadal (3-5 year) variation in sea level as determined using the EMD method; the curves are obtained by adding the five lowest-frequency intrinsic-mode functions.

The blue and red dashed lines are the linear regression fits to the data over successive 30-year periods, 19531983 and 1983-2013. They show how the rate of sea-level rise has changed over the last 60 years. The linear trends indicate that sea-level rise has accelerated at all stations to varying degrees. For the period 19531983 the rate of sea level at Townsville was $1.96 \mathrm{~mm} \mathrm{yr}^{-1}$, whereas for the period 1983-2013 the rate was $2.62 \mathrm{~mm} \mathrm{yr}^{-1}$. Similarly, at Bundaberg the rate of sea-level rise increased from $-0.948 \mathrm{~mm} \mathrm{yr}^{-1}$ in the first period to $1.8 \mathrm{~mm} \mathrm{yr}^{-1}$ in the second period. However, it should be noted that at these two stations, the data did not cover the full 60 years. In contrast, Newcastle and Sydney both have quite lengthy data records, and both indicate an increase in the rate of sea-level rise. For Newcastle the sea-level rise was $1.04 \mathrm{~mm} \mathrm{yr}^{-1}$ over the period 1953-1983, compared with $2.8 \mathrm{~mm} \mathrm{yr}^{-1}$ over the period 1983-2013. For Sydney the rate increased from $0.537 \mathrm{~mm} \mathrm{yr}^{-1}$ to $1.89 \mathrm{~mm} \mathrm{yr}^{-1}$ in the same two periods.

The observation that acceleration in sea-level rise is high near the EAC separation point suggests a possible link between changes in sea level and regional ocean dynamics such as the EAC.

It should be mentioned that the linear regression results demonstrate that the data set is non-linear and non- 
F.M. Ziaeyan Bahri et al. , Modelling sea level and EAC co-variability using HHT...

stationary, and hence the need for a more appropriate method of analysis, just as that offered by the EMD/HHT method.
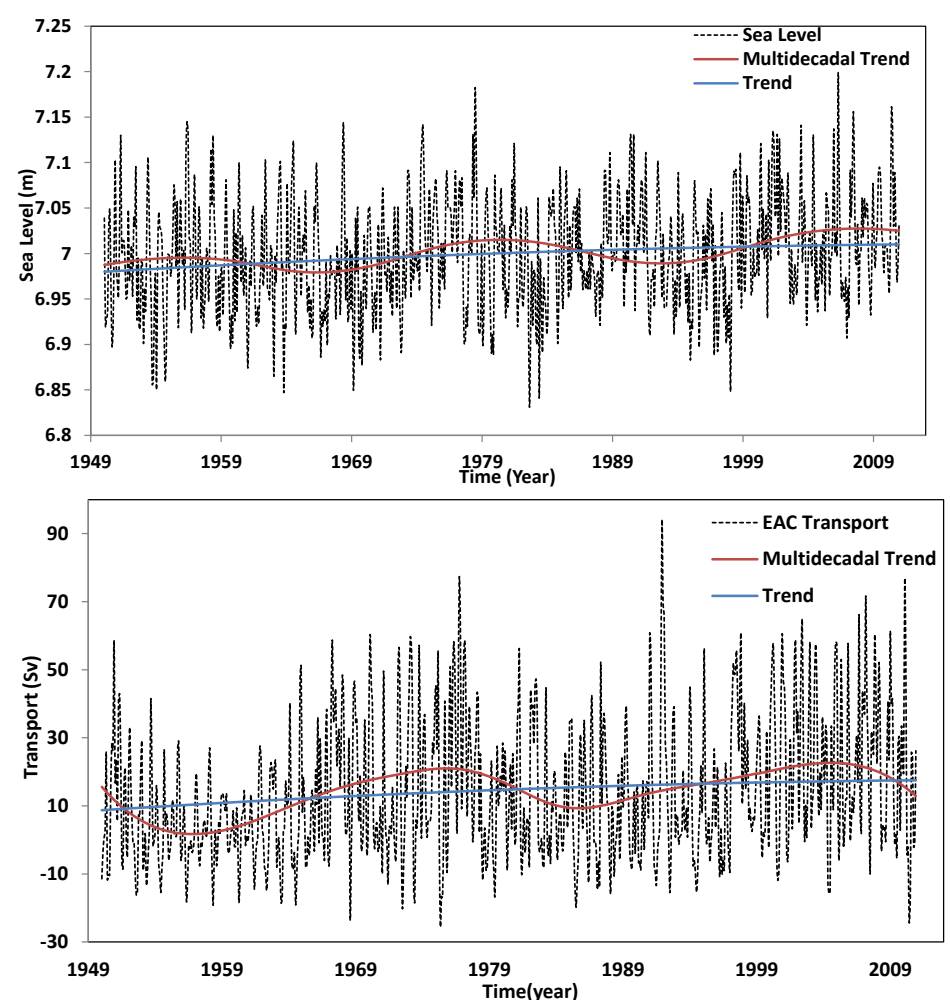

Figure 2. Sea-level and EAC variability. Red curves indicate the multidecadal trend and the blue lines are the trend

\subsection{Co-variability of EAC and sea level}

Figure 2 shows the sea-level and EAC transport time series from 1950 to 2010 at Fort Denison, Sydney. Also shown in Figure 2 are the long-term trend (blue line) given by the EMD procedure. These indicate that the sea level rises by about $0.5 \mathrm{mmyr}^{-1}$ and the EAC intensifies by about $0.15 \mathrm{Svyr}^{-1}$. The solid red curve indicate the multi-decadal trend, which was obtained from the HHT/EMD method by adding the lowest frequency mode to the trend. A degree of co-variability between EAC and sea level is apparent at the multi-decadal time scale.

Figure 3 shows the results obtained from applying EMD to the decadal oscillation and multi-decadal variations in sea level and EAC transport. The time series representing the multi-decadal sea-level and EAC variability (top panel of Figure 3) are obtained by adding the two lowest frequency IMFs (excluding the long-term trend). This produces time series with periods of approximately 25 years. The multi-decadal variability of EAC and sea level display similar patterns and a quasi-constant phase difference. According to the analyses presented, sea level is highly correlated ( $r=0.84$, with a significance of $p<10^{-4}$ ) with EAC transport lagged by 7 years.

The decadal variation in sea level and EAC can be seen in the bottom panel of Figure 3. The figure shows the fifth IMF for the sea-level and EAC time series. For sea level, the fifth IMF had a period of approximately 10 years, while for EAC the fifth IMF had a period of approximately 6 years. The decadal variation in EAC and sea level display some commensurate variations but also exhibit periods when the variations are at odds with one another. The difference in average periods of the IMFs for sea level and EAC indicate that the IMFs considered have been affected by mode mixing (Deering and Kaiser, 2005) and so the results should be considered with a degree of caution. This appears particularly to be the case in the period 1980-1990. 
F.M. Ziaeyan Bahri et al. , Modelling sea level and EAC co-variability using HHT...

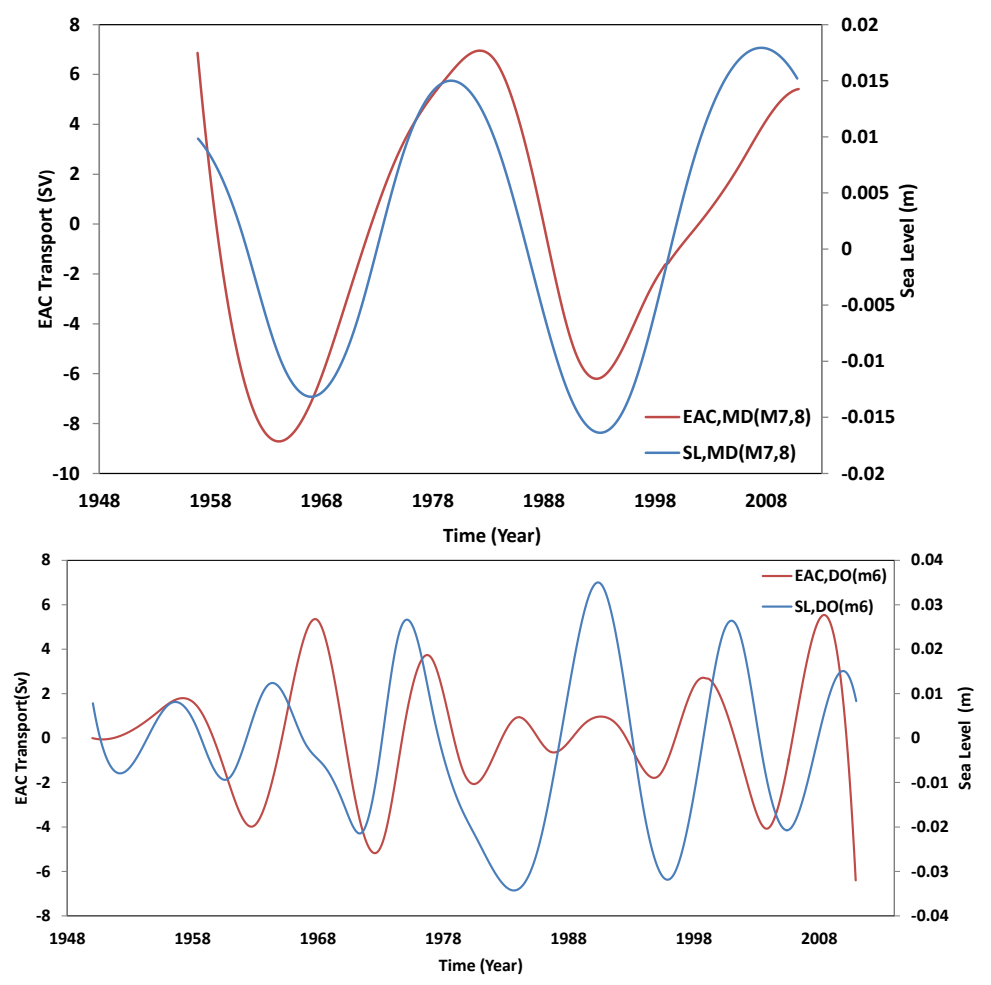

Figure 3. Sea-level and EAC Dicadal Oscilation(DO) and Multi-decadal(MD) variability

It is also possible that the apparent (lagged) covariability between multi-decadal oscillation of EAC and sea level, could be partly an artifact of the modelling methodology. Indeed, there is no obvious physical driver that would account for the 7 year lag in the two modes. This issue will be investigated in more detail in future work.

\section{CONCLUSIONS}

In this study we investigated the variability in sea level and the East Australian Current (EAC) transport using the Hilbert-Huang Transport method. A number of conclusions may be drawn from the analyses. Firstly, the similarity in the long-term trends in the monthly sea-level data over the east coast of Australia at different geographical locations suggests a common large-scale forcing. Local land subsidence, local wind patterns and local ocean dynamics may play a lesser role in variations over time scales longer than a few months.

Secondly, empirical mode decomposition of sea-level and EAC transport time series demonstrated a high correlarion $\left(r=0.84\right.$, with significant of $\left.P<10^{-4}\right)$ between EAC transport and sea level at the latitude of Fort Denison, Sydney. This co-variability was more coherent at longer time scales. Indeed, the long-term trends in the sea-level and EAC transport time series indicated a general rise in sea level and an intensification of EAC transport. At the multi-decadal time scale, EAC transport and sea level displayed significant covariability, with variations in sea level consistently lagging variations in EAC transport by about 7 years. A physical reason as to why there is such a lag is not obvious, but will be the subject of further research. Decadal variations in EAC transport and sea level at Fort Denison showed some co-variability, but the apparent presence of mode mixing across the higher-frequency IMFs made a more meaningful comparison problematic.

Moreover, at decadal to sub-decadal time scales, other driving factors such as El Niño-Southern Oscillation (ENSO) are likely to contribute to both sea-level variability and changes in EAC transport. Other such drivers (e.g. ENSO, IPO) will be incorporated into further work as part of a more comprehensive study of regional sea-level variability and ocean dynamics off eastern Australia. 
F.M. Ziaeyan Bahri et al. , Modelling sea level and EAC co-variability using HHT...

\section{REFERENCES}

Cai, W. (2006). Antarctic ozone depletion causes an intensification of the southern ocean super-gyre circulation. Geophysical Research Letters 33(3).

Cetina-Heredia, P., M. Roughan, E. Van Sebille, and M. Coleman (2014). Long-term trends in the east australian current separation latitude and eddy driven transport. Journal of Geophysical Research: Oceans 119(7), 4351-4366.

Church, J. A., J. R. Hunter, K. L. McInnes, and N. J. White (2006). Sea-level rise around the australian coastline and the changing frequency of extreme sea-level events. Australian Meteorological Magazine 55(4), 253-260.

Deering, R. and J. F. Kaiser (2005). The use of a masking signal to improve empirical mode decomposition. In Proceedings of the IEEE International Conference on Acoustics, Speech, and Signal Processing, 2005., Volume 4, pp. iv-485. IEEE.

Ezer, T., L. P. Atkinson, W. B. Corlett, and J. L. Blanco (2013). Gulf Stream's induced sea level rise and variability along the US mid-Atlantic coast. Journal of Geophysical Research: Oceans 118(2), 685-697.

Hahn, S. L. (1996). Hilbert transforms in signal processing. Artech House on Demand.

Holbrook, N. J., I. D. Goodwin, S. McGregor, E. Molina, and S. B. Power (2011). Enso to multi-decadal time scale changes in east australian current transports and fort denison sea level: Oceanic rossby waves as the connecting mechanism. Deep Sea Research Part II: Topical Studies in Oceanography 58(5), 547-558.

Huang, N. E., Z. Shen, S. R. Long, M. C. Wu, H. H. Shih, Q. Zheng, N.-C. Yen, C. C. Tung, and H. H. Liu (1998). The empirical mode decomposition and the Hilbert spectrum for nonlinear and non-stationary time series analysis. In Proceedings of the Royal Society of London A: Mathematical, Physical and Engineering Sciences, Volume 454, pp. 903-995. The Royal Society.

Huang, N. E. and Z. Wu (2008). A review on Hilbert-Huang transform: Method and its applications to geophysical studies. Reviews of Geophysics 46(2).

Hughes, L. (2003). Climate change and australia: trends, projections and impacts. Austral Ecology 28(4), $423-443$.

Levermann, A., A. Griesel, M. Hofmann, M. Montoya, and S. Rahmstorf (2005). Dynamic sea level changes following changes in the thermohaline circulation. Climate Dynamics 24(4), 347-354.

Masumoto, Y., H. Sasaki, T. Kagimoto, N. Komori, A. Ishida, Y. Sasai, T. Miyama, T. Motoi, H. Mitsudera, K. Takahashi, et al. (2004). A fifty-year eddy-resolving simulation of the world ocean: Preliminary outcomes of ofes (ogcm for the earth simulator). J. Earth Simulator 1, 35-56.

Mitchell, W., J. Chittleborough, B. Ronai, and G. Lennon (2000). Sea level rise in australia and the pacific. The South Pacific Sea Level and Climate Change Newsletter, Quarterly Newsletter 5, 10-19.

Rahmstorf, S. (2012a). Modeling sea level rise. Nature Education Knowledge 3(3), 4.

Rahmstorf, S. (2012b). Sea-level rise: towards understanding local vulnerability. Environmental Research Letters 7(2), 021001.

Ridgway, K., R. Coleman, R. Bailey, and P. Sutton (2008). Decadal variability of east australian current transport inferred from repeated high-density xbt transects, a ctd survey and satellite altimetry. Journal of Geophysical Research: Oceans (1978-2012) 113(C8).

Sasaki, H., M. Nonaka, Y. Masumoto, Y. Sasai, H. Uehara, and H. Sakuma (2008). An eddy-resolving hindcast simulation of the quasiglobal ocean from 1950 to 2003 on the earth simulator. In High resolution numerical modelling of the atmosphere and ocean, pp. 157-185. Springer.

Schlurmann, T. (2002). Spectral analysis of nonlinear water waves based on the Hilbert-Huang transformation. Journal of Offshore Mechanics and Arctic Engineering 124(1), 22-27.

Sebille, E., M. H. England, J. D. Zika, and B. M. Sloyan (2012). Tasman leakage in a fine-resolution ocean model. Geophysical Research Letters 39(6). 